\title{
Switch from Tenofovir Disoproxil Fumarate to Tenofovir Alafenamide in People Living with HIV: Lipid Changes and Statin Underutilization
}

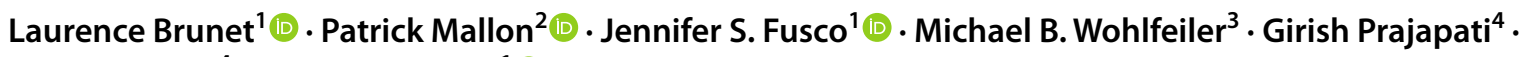 \\ Andrew Beyer ${ }^{4}$. Gregory P. Fusco ${ }^{1}$ D
}

Accepted: 2 September 2021 / Published online: 21 September 2021

(c) The Author(s) 2021

\begin{abstract}
Background and Objective Many people living with HIV (PLWH) on stable tenofovir disoproxil fumarate (TDF)-containing regimens have switched to tenofovir alafenamide (TAF), despite the potential lipid-lowering effect of TDF. We aimed to assess the impact of switching from TDF to TAF on lipids in real-world clinical practice.

Methods PLWH prescribed TDF for $\geq 4$ weeks who switched to TAF were identified in the OPERA cohort. Patterns of dyslipidemia were compared before and after switch based on NCEP ATPIII guidelines. Elevated 10-year risk of atherosclerotic cardiovascular disease (ASCVD $\geq 7.5 \%$ ) and statin use were assessed.

Results Among 6423 PLWH switched from TDF to TAF, the proportion with dyslipidemia/severe dyslipidemia observed after switch from TDF to TAF increased statistically significantly $(p<0.0001)$ with total cholesterol $(5-10 \%)$, low-density lipoprotein cholesterol (16-23\%), and triglycerides (21-27\%), but decreased statistically significantly with high-density lipoprotein cholesterol $(35-30 \%, p<0.0001)$. These patterns of dyslipidemia persisted in sensitivity analyses restricted to PLWH who maintained all other antiretrovirals $(N=4328)$ or stratified by pharmaco-enhancer use before and after switch. An elevated ASCVD risk was detected in 29\% before and 31\% after switch. As many as 59\% of PLWH with an elevated ASCVD risk were not prescribed a statin after switch from TDF to TAF.

Conclusions In this large, diverse population of PLWH in the USA, the switch from TDF to TAF was associated with development of less favorable lipid profiles, regardless of pharmaco-enhancers or third-agent use. Statins remained underutilized after a switch from TDF to TAF.
\end{abstract}

Laurence Brunet

laurence.brunet@epividian.com

1 Epividian, Inc., 4819 Emperor Blvd., Suite 400, Durham, NC 27703, USA

2 Centre for Experimental Pathogen Host Research, University College Dublin, Dublin, Ireland

3 AIDS Healthcare Foundation, Miami, FL, USA

4 Merck \& Co., Inc., Kenilworth, NJ, USA

\section{Key Points}

Among 6423 people living with HIV, switching from TDF to TAF led to less favorable lipid profiles, with statistically significant increases in total cholesterol, lowdensity lipoprotein cholesterol, and triglycerides, and a statistically significant decrease in high-density lipoprotein cholesterol.

Similar patterns emerged when controlling for pharmaco-enhancers and third agents.

Statins remained underutilized after a switch from TDF to TAF. 


\section{Introduction}

Tenofovir disoproxil fumarate (TDF) and tenofovir alafenamide (TAF) are among the antiretrovirals (ARVs) recommended as part of the backbone of antiretroviral therapy (ART) by the US Department of Health and Human Services HIV treatment guidelines [1]. Use of TDF has been associated with increased risk of renal tubular dysfunction [2,3], and greater reductions in bone mineral density at treatment initiation [3, 4]. However, in a meta-analysis of 14 clinical trials, no difference was found in bone markers or renal tubular events, overall, although a small, statistically significant increase in renal adverse events-related discontinuations was detected with a pharmacokinetic (PK) boosting agent only [5].

Nevertheless, there is also evidence that TDF could have an independent lipid-lowering effect [3]. Statistically significant decreases in fasting total cholesterol (CHOL) and low-density lipoprotein (LDL) have been observed with TDF compared to placebo in small trials [6-8]. Improvement in lipid profiles have also been described with TDF compared to other agents in clinical trials $[9,10]$ and observational studies [11]. Such improvements were also observed in other populations such as HIV-negative men on pre-exposure prophylaxis (PrEP) with TDF/FTC [12] and individuals with chronic hepatitis B treated with TDF [13].

Nonetheless, many people living with HIV (PLWH) on stable TDF-containing regimens have been switched to TAF to avoid potential bone and renal toxicity [14]. The impact of switching directly from TDF to TAF on lipids has been explored in a few randomized controlled trials (RCTs) [15-17] and small observational studies [18-22]. While these studies showed small but statistically significant increases in lipid levels after switching away from TDF at a population level, the clinical relevance of these changes, specifically in relation to risk of cardiovascular disease, remains unclear, as does the real-world impact of removing TDF on lipids in clinical practice.

For primary prevention of atherosclerotic cardiovascular disease (ASCVD), the American College of Cardiology and the American Heart Association (ACC/AHA) guidelines recommend initiation of HMG co-enzyme A reductase inhibitors (statins) for individuals with an elevated 10 -year ASCVD risk ( $\geq 7.5 \%$ ) [23]. Current HIV treatment guidelines recommend a random or fasting lipid profile be performed yearly, as well as at entry into care and at ART initiation or modification [24]. Thus, failure to initiate statins in PLWH with an ASCVD $\geq 7.5 \%$ at switch may represent a missed opportunity for primary prevention.

The objective of this study was to compare lipid profiles among PLWH before and after a switch from TDF to TAF. More specifically, we aimed to compare dyslipidemia and lipid levels before and after a switch from TDF to TAF, to describe ASCVD risk before and after switch, and to identify missed opportunities for statin prescription around the time of switch from TDF to TAF.

\section{Methods}

\subsection{Study Population}

The Observational Pharmacoepidemiology Research and Analysis $\left(\right.$ OPERA $\left.^{\circledR}\right)$ cohort is a clinical cohort utilizing prospectively collected, electronic medical record (EMR) data. As of October 23, 2018, the OPERA cohort included 93170 PLWH in care attending 84 out-patient clinics in 18 US states and territories. All data reflect routine medical care, with visits and testing scheduled at the discretion of the treating providers. Information captured in the EMR system at each site is retrieved, cleaned, aggregated, and stripped of identifiers to maintain patient confidentiality.

The study population consisted of PLWH aged at least 18 years, exposed to a TDF-containing regimen for a minimum of 4 weeks, who switched to a TAF-containing regimen between November 5, 2015, and March 31, 2018. Those PLWH included in the analysis had at least one lipid panel while on TDF within 6 months prior to switch and at least one lipid panel at any time after switch to TAF. Excluded from the study population were individuals who received a TDF-containing regimen for post-exposure prophylaxis (PEP) or pre-exposure prophylaxis (PrEP), PLWH with > 45 days between discontinuation of TDF and initiation of TAF, and PLWH who received TDF and/or TAF through a clinical trial. PLWH included in the study were observed from the date of switch until the first of the following censoring events: (1) discontinuation of TAF, (2) 12 months after the last clinical contact, (3) death, or (4) June 30, 2018 (study end).

\subsection{Measurements}

Laboratory values for total cholesterol (CHOL), LDL, high-density lipoprotein (HDL) and triglycerides (TG) were obtained from the EMR data. The last lipid panel performed while on TDF and the first lipid panel performed at least seven days after switch to TAF were selected; in the absence of pharmacy records, this cut-off was selected to allow PLWH time to fill their prescription and begin TAF use before lipids were measured. Lipid values were categorized according to the NCEP ATPIII guidelines (Table 1) [25].

The 10-year risk of ASCVD was derived from the Pooled Cohort Equations calculator [26]. The resulting ASVCD score predicts the risk of developing a first ASCVD event (i.e. nonfatal myocardial infarction, coronary heart disease death, or fatal or nonfatal stroke) over 10 years among people free from 
Table 1 NCEP ATPIII categorization of lipids [25]

\begin{tabular}{lllll}
\hline Category & Total cholesterol & Low-density lipoprotein & High-density lipoprotein & Triglycerides \\
\hline Normal & $<200 \mathrm{mg} / \mathrm{dL} ;<5.17 \mathrm{mmol} / \mathrm{L}$ & $<100 \mathrm{mg} / \mathrm{dL} ; 2.59 \mathrm{mmol} / \mathrm{L}$ & $\geq 60 \mathrm{mg} / \mathrm{dL} ; \geq 1.55 \mathrm{mmol} / \mathrm{L}$ & $<150 \mathrm{mg} / \mathrm{dL} ;<1.69 \mathrm{mmol} / \mathrm{L}$ \\
Borderline & $\geq 200$ to $<240 \mathrm{mg} / \mathrm{dL} ;$ & $\geq 100$ to $<130 \mathrm{mg} / \mathrm{dL} ;$ & $\geq 40$ to $<60 \mathrm{mg} / \mathrm{dL} ; \geq 1.03$ & $(\geq 150 \mathrm{to}<200 \mathrm{mg} / \mathrm{dL} ; \geq 1.69$ \\
abnormal & $\geq 5.17$ to $<6.21 \mathrm{mmol} / \mathrm{L}$ & $\geq 2.59$ to $<3.36 \mathrm{mmol} / \mathrm{L}$ & to $<1.55 \mathrm{mmol} / \mathrm{L}$ & to $<2.26 \mathrm{mmol} / \mathrm{L}$ \\
Dyslipidemia & $\geq 240$ to $<280 \mathrm{mg} / \mathrm{dL} ;$ & $\geq 130$ to $<160 \mathrm{mg} / \mathrm{dL} ;$ & $<40 \mathrm{mg} / \mathrm{dL} ;<1.03 \mathrm{mmol} / \mathrm{L}$ & $\geq 200$ to $<500 \mathrm{mg} / \mathrm{dL} ; \geq 2.26$ \\
& $\geq 6.21$ to $<7.24 \mathrm{mmol} / \mathrm{L}$ & $\geq 3.36$ to $<4.14 \mathrm{mmol} / \mathrm{L}$ & to $<5.65 \mathrm{mmol} / \mathrm{L}$ \\
$\begin{array}{ll}\text { Severe/very } \\
\begin{array}{l}\text { severe dyslipi- } \\
\text { demia }\end{array}\end{array}$ & $280 \mathrm{mg} / \mathrm{dL} ; \geq 7.24 \mathrm{mmol} / \mathrm{L} \geq 160 \mathrm{mg} / \mathrm{dL} ; \geq 4.14 \mathrm{mmol} / \mathrm{L}$ & $\mathrm{NA}$ & $\geq 500 \mathrm{mg} / \mathrm{dL} ; \geq 5.65 \mathrm{mmol} / \mathrm{L}$ \\
\hline
\end{tabular}

NCEP ATPIII National Cholesterol Education Program Adult Treatment Panel III

ASCVD [26]. The ACC/AHA recommend the initiation of statin therapy as primary prevention for ASCVD risk reduction among persons with ASCVD scores $\geq 7.5 \%$ [23]. ASCVD scores were calculated based on each person's sex, age, race, CHOL, HDL, systolic blood pressure, treatment for high blood pressure, diabetes, and smoking status. The ASCVD score algorithm was developed among those aged $40-79$ years, with systolic blood pressure 90-200 mmHg, HDL 20-100 mg/dL, and CHOL 130-320 mg/dL [26]. ASCVD scores for PLWH with values outside of these specified ranges were imputed using the lower or upper bound, as appropriate [27]. In this analysis, ASCVD scores were calculated at the time of the last lipid panel on TDF and at the time of the first lipid panel on TAF, at least seven days after the switch from TDF to TAF.

ART and non-ART medication prescriptions were retrieved from the EMR. The presence of a PK boosting agent (ritonavir or cobicistat) or statin prescription was assessed at the time of the last lipid panel on TDF. Postswitch, the presence of PK boosting agent was assessed at the time of the first lipid panel at least seven days after the switch from TDF to TAF. Prescription of a statin was assessed at any time on or after the first lipid panel on TAF.

\subsection{Analyses}

The median value and interquartile range (IQR) for each lipid was described before and after switch, with Wilcoxon Rank Sum tests used for statistical comparison. The percentage change in each lipid level was averaged across individuals and $95 \%$ confidence intervals (CI) for the mean percent change were obtained based on the normal distribution. The proportion of PLWH within each NCEP ATPIII dyslipidemia category was also calculated and compared before and after switch using Pearson's chi-square test. In addition to performing these analyses on the overall population, a sensitivity analysis was conducted on the sub-population of PLWH who did not change any other component of their ART regimen apart from a switch from TDF to TAF. An analysis stratified by boosting agent use (ritonavir or cobicistat) before and after switch was also performed. Finally,
Table 2 Demographic and clinical characteristics at the time of switch from TDF to TAF $(N=6451)$

\begin{tabular}{ll}
\hline Characteristic & $n(\%)$ \\
\hline Age, years & \\
Median (IQR) & $47.9(37.9,54.7)$ \\
$18-25$ & $228(3.5)$ \\
$26-49$ & $3454(53.5)$ \\
$50+$ & $2769(42.9)$ \\
Female & $1010(15.7)$ \\
African American & $2126(33.0)$ \\
Hispanic & $1870(29.0)$ \\
Geographic location & \\
South & $3577(55.5)$ \\
West & $2107(32.7)$ \\
Northeast & $631(9.8)$ \\
Midwest & $135(2.1)$ \\
HIV viral load $<50$ copies/mL & $5305(82.2)$ \\
Median CD4 cell count, cells/ $\mu \mathrm{L}(\mathrm{IQR})$ & $635(452,845)$ \\
Diabetes mellitus & $246(3.8)$ \\
Hypertension & $429(6.7)$ \\
HCV co-infection & $369(5.7)$ \\
Median months on TDF pre-switch (IQR) & $29.2(13.8,51.5)$ \\
\hline
\end{tabular}

$I Q R$ interquartile range, $n$ number, $T A F$ tenofovir alafenamide, TDF tenofovir disoproxil fumarate

among those for whom the ASCVD risk could be calculated, the proportion of PLWH with an elevated ASCVD risk was estimated before and after switch; the proportion of PLWH prescribed statins before and after switch was also assessed, stratified by 10 -year ASCVD risk.

\section{Results}

\subsection{Study Population}

A total of 6451 PLWH who switched from TDF to TAF met all inclusion criteria, with a first post-switch lipid panel performed at a median of 3.9 months after switch from TDF 
Table 3 Clinical characteristics at the last lipid panel on TDF and at the first lipid panel on TAF $(N=6451)$

\begin{tabular}{lll}
\hline Characteristic & $\begin{array}{l}\text { Last lipid panel on } \\
\text { TDF, } n(\%)\end{array}$ & $\begin{array}{l}\text { First lipid } \\
\text { panel on TAF, } \\
n(\%)\end{array}$ \\
\hline $\begin{array}{l}\text { Anchor agent class } \\
\text { PI }\end{array}$ & $1566(24.3)$ & $1228(19.0)$ \\
NNRTI & $2319(35.9)$ & $1546(24.0)$ \\
INSTI & $3007(46.6)$ & $4185(64.9)$ \\
Other & $46(0.7)$ & $41(0.6)$ \\
More than one anchor agent & $527(8.2)$ & $563(8.7)$ \\
Boosting agent used & $3292(51.0)$ & $3998(61.8)$ \\
Imputed ASCVD risk score & & \\
$<7.5 \%$ & $3939(61.1)$ & $3877(60.1)$ \\
$\geq 7.5 \%$ & $1862(28.9)$ & $2004(31.1)$ \\
Missing & $650(10.1)$ & $570(8.8)$ \\
\hline
\end{tabular}

ASCVD atherosclerotic cardiovascular disease, INSTI integrase strand transfer inhibitor, $n$ number, NNRTI non-nucleoside reverse transcriptase inhibitor, $P I$ protease inhibitor, $T A F$ tenofovir alafenamide, $T D F$ tenofovir disoproxil fumarate

${ }^{a}$ ASCVD risk score calculated based on sex, age, race, total cholesterol, HDL, systolic blood pressure, hypertension treatment, diabetes, and smoking status; imputed using the limit value if out of range

to TAF (IQR: 2.7, 6.7). The main population included $16 \%$ female and 33\% African American PLWH; most had wellcontrolled HIV, and few had comorbid conditions (Table 2). At the last lipid panel on TDF, 24\% were on an ARV regimen containing a protease inhibitor (PI) and $51 \%$ were on a boosted regimen. At the first lipid panel post-switch to TAF, $19 \%$ were on a PI and $62 \%$ on a boosted regimen (Table 3); the most common third agents used are listed in Table 4. Among 4328 PLWH who maintained all other ARVs, 18\% were on a PI and 58\% were on a boosted regimen.

\subsection{Changes in Lipids Before and After Switch}

In the main study population, the first lipid panel occurred a median of 3.9 months (IQR: 2.7, 6.7) after the switch from TDF to TAF; $60 \%$ occurred 3-6 months after switch and $30 \%$ occurred $>6$ months after switch. All lipid levels increased after switching from TDF to TAF. The mean percentage increase in lipids observed was most pronounced with TG $(+23.8 \%)$, followed by LDL $(+11.1 \%)$, but less pronounced with CHOL $(+7.9 \%)$ and $\mathrm{HDL}(+7.1 \%$; Table 5). Median lipid levels also increased statistically significantly for all lipids assessed after the switch from TDF to TAF (Table 5). Similar patterns were observed among PLWH who only switched from TDF to TAF while maintaining all other ARVs, although point estimates for the mean percentage changes were slightly higher (Table 5).

In the main study population, differences in the distribution of dyslipidemia categories before and after switch were statistically significant for all lipids (Fig. 1A). The proportion of PLWH with dyslipidemia or severe dyslipidemia increased after switching from TDF to TAF for CHOL, LDL and TG, while the proportion with dyslipidemia decreased for HDL, resulting in an overall increase in the proportion of PLWH with dyslipidemia or severe dyslipidemia as measured with CHOL $(p<0.0001)$, LDL $(p<0.0001)$ and TG $(p<0.0001$; Fig. 1A). Similar patterns were observed in PLWH who maintained all other ARVs (Fig. 1B).

\subsection{Changes in Lipids Before and After Switch, by Use of Boosting Agent}

After stratification of the main study population by boosting agent use before and after switch, the overall patterns of dyslipidemia categories were similar to those in the entire population. However, among the 165 PLWH on a boosted

Table 4 Most common ART regimen before and after switch $(N=6451)$

\begin{tabular}{llll}
\hline $\begin{array}{l}\text { Third agent in TDF-containing regi- } \\
\text { mens }\end{array}$ & $n(\%)$ & Third agent in TAF-containing regimens & \\
\hline Elvitegravir/cobicistat & $1757(27.2)$ & Elvitegravir/cobicistat & $2743(42.5)$ \\
Rilpivirine & $955(14.8)$ & Rilpivirine & $1187(18.4)$ \\
Efavirenz & $910(14.1)$ & Dolutegravir & $674(10.4)$ \\
Dolutegravir & $524(8.1)$ & Darunavir/cobicistat & $425(6.6)$ \\
Darunavir/ritonavir & $411(6.4)$ & Raltegravir & $195(3.0)$ \\
Darunavir/cobicistat & $282(4.4)$ & Darunavir/ritonavir & $183(2.8)$ \\
Raltegravir & $281(4.4)$ & Nevirapine & $155(2.4)$ \\
Atazanavir/ritonavir & $267(4.1)$ & Dolutegravir + darunavir/cobicistat & $135(2.1)$ \\
Nevirapine & $225(3.5)$ & Atazanavir/ritonavir & $96(1.5)$ \\
Atazanavir/cobicistat & $72(1.1)$ & Atazanavir/cobicistat & $89(1.4)$ \\
Other & $766(11.9)$ & Other & $569(8.8)$ \\
\hline
\end{tabular}

$A R T$ antiretroviral therapy, $n$ number, $T A F$ tenofovir alafenamide, $T D F$ tenofovir disoproxil fumarate 
Table 5 Absolute and relative change in lipids pre- versus post-switch from TDF to TAF among all PLWH and among PLWH who maintained all other ARVs

\begin{tabular}{|c|c|c|c|c|}
\hline Parameter & $\begin{array}{l}\text { Pre-switch (TDF) lipid lev- } \\
\text { els (mg/dL), median (IQR) }\end{array}$ & $\begin{array}{l}\text { Post-switch (TAF) lipid lev- } \\
\text { els (mg/dL), median (IQR) }\end{array}$ & $p$-value ${ }^{\mathrm{a}}$ & $\begin{array}{l}\text { Mean percentage } \\
\text { change }(95 \% \mathrm{CI})\end{array}$ \\
\hline \multicolumn{5}{|c|}{ All PLWH switching from TDF to TAF $(N=6451)$} \\
\hline CHOL & $174(151,199)$ & $184(159,211)$ & $<0.0001$ & $7.9(7.4,8.3)$ \\
\hline LDL & $97(77,118)$ & $104(82,127)$ & $<0.0001$ & $11.1(9.2,12.9)$ \\
\hline HDL & $45(36,55)$ & $46(38,57)$ & $<0.0001$ & $7.1(6.2,8.0)$ \\
\hline TG & $127(88,186)$ & $139(94,207)$ & $<0.0001$ & $23.8(22.0,25.5)$ \\
\hline \multicolumn{5}{|c|}{ PLWH switching from TDF to TAF without other ARV changes $(N=4328)$} \\
\hline CHOL & $171(148,196)$ & $183(158,211)$ & $<0.0001$ & $9.0(8.5,9.6)$ \\
\hline LDL & $96(76,116)$ & $104(82,127)$ & $<0.0001$ & $12.2(9.6,14.8)$ \\
\hline HDL & $44(36,54)$ & $46(38,57)$ & $<0.0001$ & $8.1(6.9,9.2)$ \\
\hline TG & $124(87,183)$ & $138(94,208)$ & $<0.0001$ & $25.8(23.7,28.0)$ \\
\hline
\end{tabular}

$A R V$ antiretroviral, $C H O L$ total cholesterol, $H D L$ high-density lipoprotein, $L D L$ low-density lipoprotein, $n$ number, $P L W H$ people living with HIV, TAF tenofovir alafenamide, $T D F$ tenofovir disoproxil fumarate, $T G$ triglyceride

${ }^{\mathrm{a}} p$-value for the comparison of lipid value distribution pre- versus post-switch

Fig. 1 Lipid profiles pre- and post-switch among A all PLWH $(N=6451)$ and B PLWH who maintained all other ARVs sensitivity analysis $(N=4328)$. $A R V$ antiretroviral, $C H O L$ total cholesterol, $H D L$ high-density lipoprotein, $L D L$ low-density lipoprotein, $n$ number, $P L W H$ people living with HIV, TAF tenofovir alafenamide, $T D F$ tenofovir disoproxil fumarate, $T G$ triglyceride. * $p$-value for the pre-post switch comparison $<0.0001$
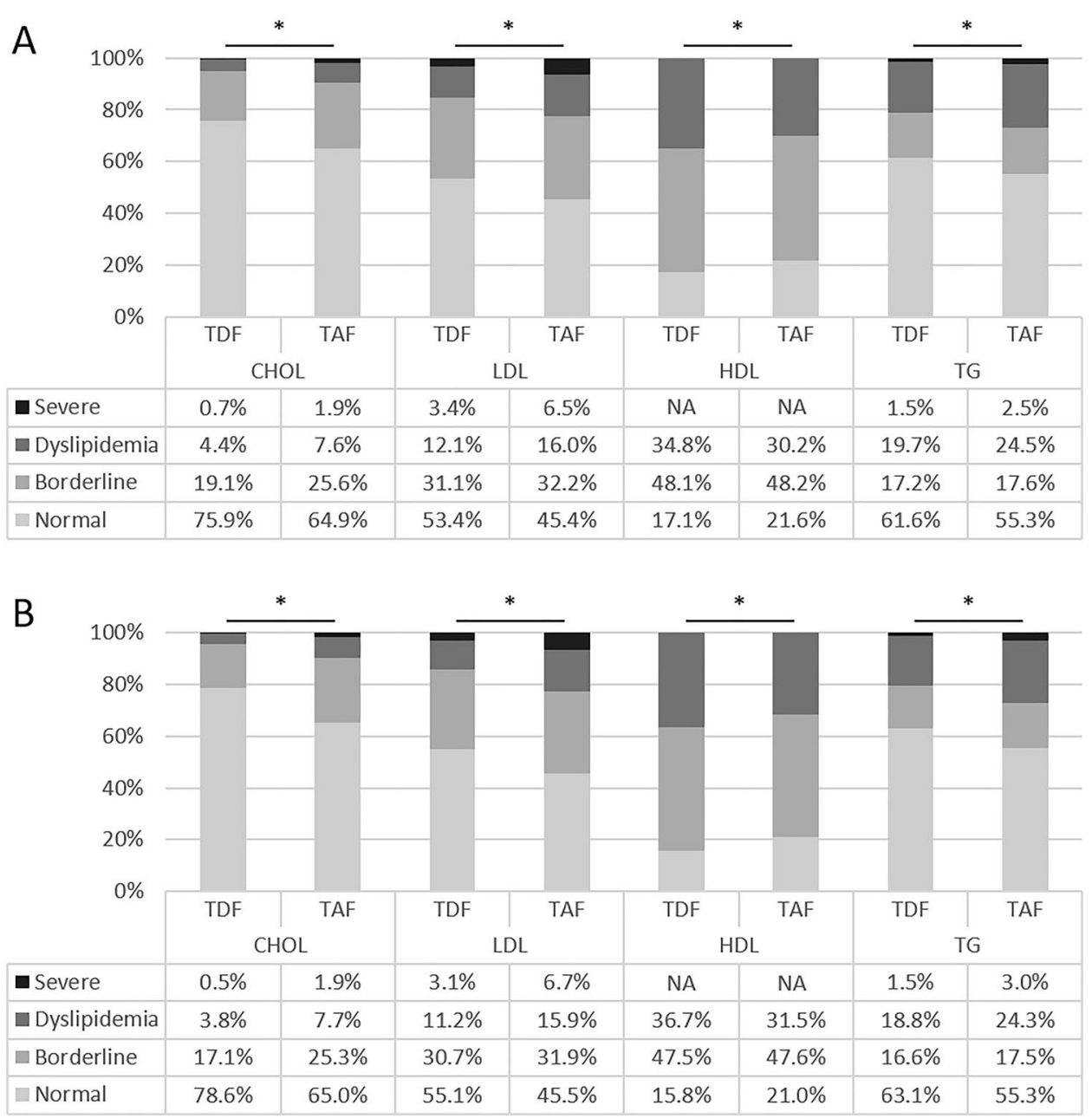
Fig. 2 Lipid profiles by boosting agent use A pre-switch ( $n=$ 165), B post-switch $(n=861)$, C pre- and post-switch $(n=$ $3126)$, or D never $(n=2298)$. CHOL total cholesterol, $H D L$ high-density lipoprotein, $L D L$ low-density lipoprotein, $n$ number, $P L W H$ people living with HIV, TAF tenofovir alafenamide, $T D F$ tenofovir disoproxil fumarate, $T G$ triglyceride. * $p$-value for the pre-post switch comparison $<0.05$
A Boosted pre-switch

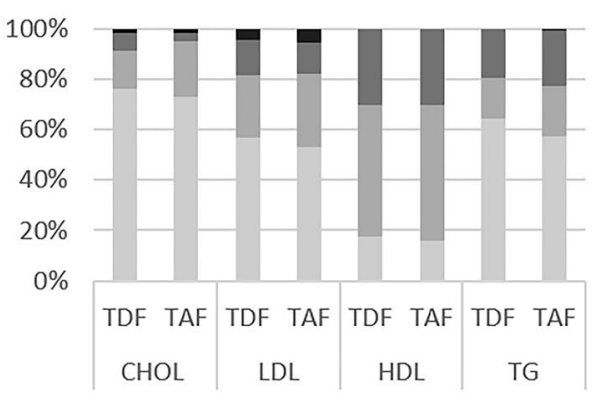

C Boosted pre- \& post-switch

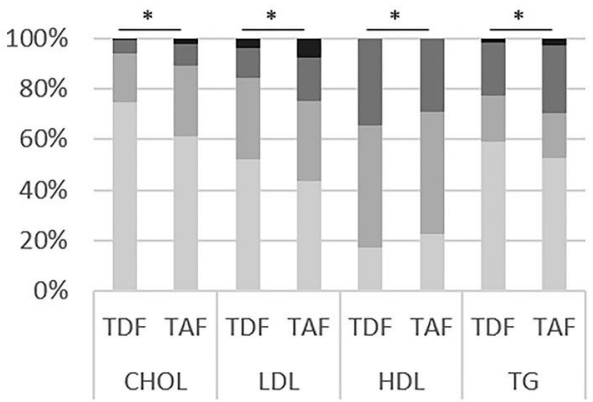

B Boosted post-switch

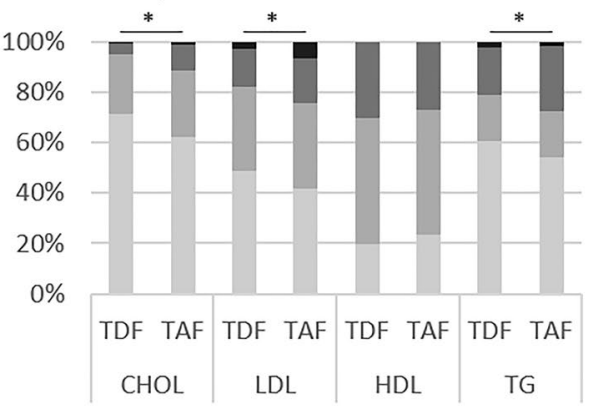

D Never boosted

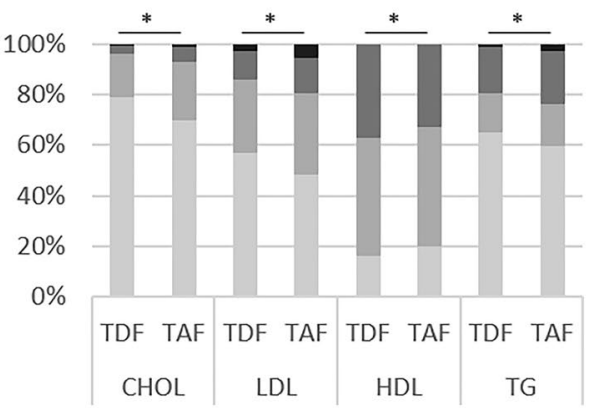

Table 6 Absolute and relative change in lipids pre- versus post-switch among all PLWH, stratified by boosting agent use

\begin{tabular}{|c|c|c|c|c|}
\hline Parameter & $\begin{array}{l}\text { Pre-switch (TDF) lipid lev- } \\
\text { els }(\mathrm{mg} / \mathrm{dL}) \text {, median (IQR) }\end{array}$ & $\begin{array}{l}\text { Post-switch (TAF) lipid lev- } \\
\text { els (mg/dL), median (IQR) }\end{array}$ & $p$-value & $\begin{array}{l}\text { Mean percentage } \\
\text { change }(95 \% \mathrm{CI})\end{array}$ \\
\hline \multicolumn{5}{|c|}{ Boosted pre-switch only $(n=165)$} \\
\hline CHOL & $174(140,197)$ & $173(146,203)$ & 0.6503 & $2.3(-0.6,5.2)$ \\
\hline LDL & $95(72,118)$ & $98(74,119)$ & 0.4518 & $5.1(0.1,10.1)$ \\
\hline HDL & $45(39,54)$ & $45(38,55)$ & 0.9425 & $7.2(-0.4,14.8)$ \\
\hline TG & $127(87,183)$ & $128(88,192)$ & 0.7987 & $15.6(4.6,26.7)$ \\
\hline \multicolumn{5}{|c|}{ Boosted post-switch only $(n=861)$} \\
\hline CHOL & $180(157,204)$ & $188(165,213)$ & $<0.0001$ & $7.6(6.3,9.0)$ \\
\hline LDL & $101(80,121)$ & $106(86,128)$ & 0.0005 & $10.3(8.0,12.7)$ \\
\hline HDL & $46(38,56)$ & $47(39,59)$ & 0.0804 & $5.9(4.2,7.6)$ \\
\hline TG & $129(89,184)$ & $145(98,212)$ & 0.0002 & $25.4(20.8,29.9)$ \\
\hline \multicolumn{5}{|c|}{ Boosted pre- $\&$ post-switch $(n=3126)$} \\
\hline CHOL & $176(152,200)$ & $188(161,215)$ & $<0.0001$ & $8.8(8.1,9.5)$ \\
\hline LDL & $98(77,119)$ & $106(84,129)$ & $<0.0001$ & $13.3(9.7,17.0)$ \\
\hline HDL & $45(36,55)$ & $47(38,58)$ & $<0.0001$ & $7.7(6.4,9.1)$ \\
\hline TG & $132(92,193)$ & $145(99,215)$ & $<0.0001$ & $24.4(21.9,26.9)$ \\
\hline \multicolumn{5}{|c|}{ Never boosted $(n=2298)$} \\
\hline CHOL & $168(147,194)$ & $179(154,205)$ & $<0.0001$ & $7.0(6.3,7.8)$ \\
\hline LDL & $95.0(76,115)$ & $101(79,123)$ & $<0.0001$ & $8.8(7.5,10.0)$ \\
\hline HDL & $44(36,54)$ & $45(37,56)$ & 0.0002 & $6.7(5.3,8.1)$ \\
\hline TG & $120(85,176)$ & $129(89,196)$ & $<0.0001$ & $22.9(20.0,25.8)$ \\
\hline
\end{tabular}

$C H O L$ total cholesterol, $H D L$ high-density lipoprotein, $L D L$ low-density lipoprotein, $n$ number, $P L W H$ people living with HIV, TAF tenofovir alafenamide, $T D F$ tenofovir disoproxil fumarate, $T G$ triglyceride 
Fig. 3 Statin use by ASCVD risk score pre- and post-switch ${ }^{\mathrm{a}}$, among all PLWH. ASCVD atherosclerotic cardiovascular disease, $n$ number; $P L W H$ people living with HIV, TAF tenofovir alafenamide, $T D F$ tenofovir disoproxil fumarate.

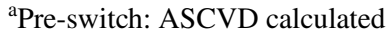
$\leq 6$ months before the last lipid panel on TDF; statin prescription at or after the last lipid panel on TDF; Post-switch: ASCVD calculated $\leq 6$ months before the first lipid panel on TAF; statin prescription at or after the first lipid panel on TAF

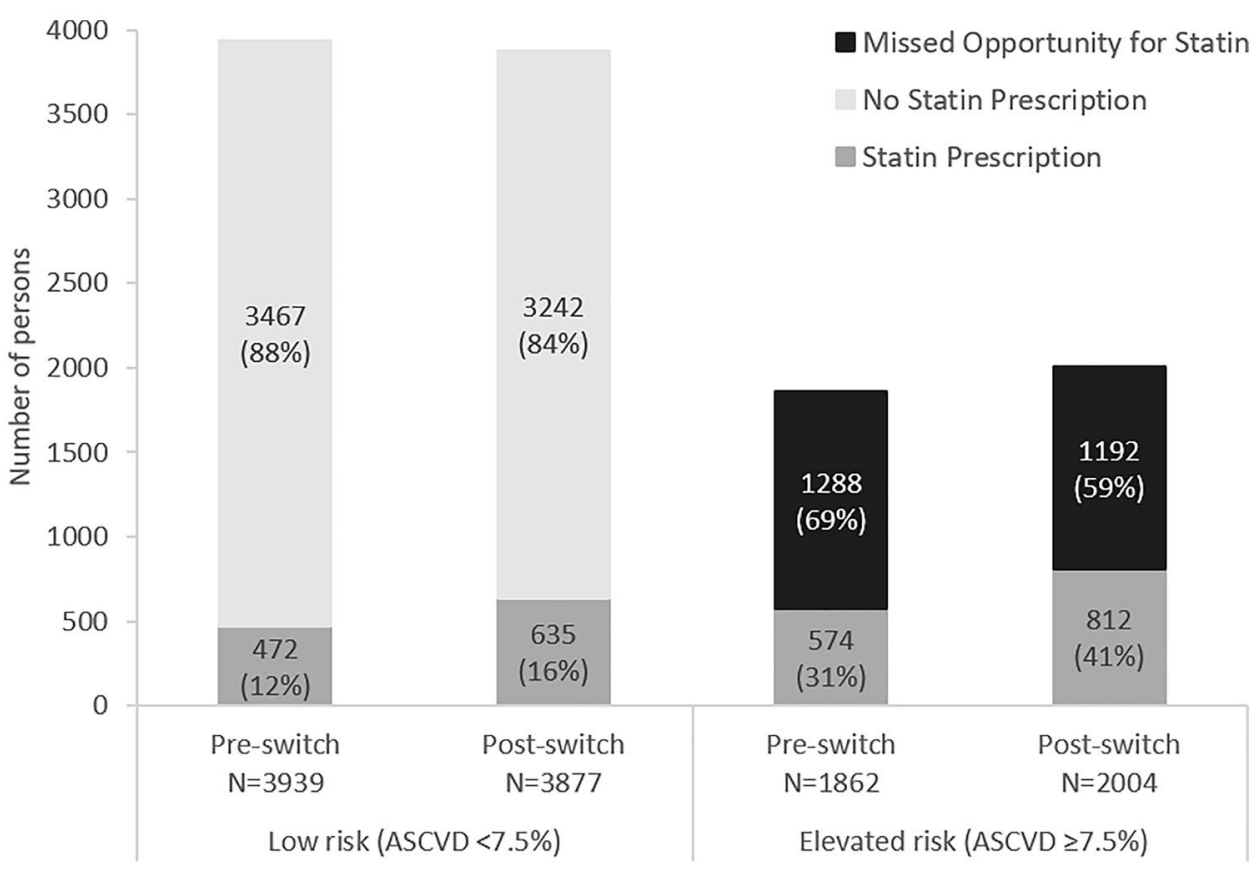

regimen prior to switch and an unboosted regimen after switch, there was no statistically significant difference in the distribution of dyslipidemia categories before and after switch (Fig. 2A), but a statistically significant mean percentage increase was observed with LDL and TG only (Table 6). Among 861 PLWH on an unboosted regimen prior to switch, and a boosted regimen after switch, statistically significant increases in dyslipidemia measured with CHOL, LDL and TG were observed after switch (Fig. 2B); mean percentage increases were statistically significant for all lipids (Table 6). Finally, among the 3126 PLWH who remained on a boosted regimen before and after switch, and among the $2298 \mathrm{PLWH}$ who remained on an unboosted regimen before and after switch, differences in the distribution of dyslipidemia categories were statistically significant for all lipids (Fig. 3C, D). Statistically significant increases in mean percentage increases in CHOL, LDL, HDL, and TG of similar magnitude to those described in the entire population were also observed (Table 6).

\subsection{ASCVD Risk and Statin Use}

After imputation, over $90 \%$ of the study population had a 10-year ASCVD risk estimated either before or after switch (Table 3). In this subset, the proportion of individuals with elevated ASCVD risk ( $\geq 7.5 \%)$ was slightly higher after switch to TAF (34\% on TAF vs $32 \%$ on TDF). Among PLWH with an elevated 10-year ASCVD risk, there was a $10 \%$ increase in the proportion of statin use, from $31 \%$ before to $41 \%$ after switching from TDF to TAF ( $p<0.0001$, Fig. 3). However, this represents considerable missed opportunities for ASCVD prevention, as 59\% of those with an elevated ASCVD risk were not prescribed statins at any point on or after their first lipid panel after switch (Fig. 3). Comparable results were obtained among PLWH who did not require ASCVD score imputation (data not shown).

\section{Discussion}

In this large, diverse cohort of PLWH in care in the USA, switching from TDF to TAF was associated with worsening CHOL, LDL and TG. The observed development of less favorable lipid profiles was not driven by changes in other ARVs as part of the switch, as almost identical changes in lipids were observed in an analysis restricted to PLWH who maintained all other ARVs, nor was it driven by use of pharmacological boosting agents, as demonstrated in stratified analysis. In addition, while switching from TDF to TAF was associated with a $10 \%$ increase in statin use among PLWH with high ASCVD risk, 59\% of PLWH with an elevated risk of ASCVD were still not prescribed statins after switch.

The magnitude of lipid increases observed in this study (CHOL: $+7.9 \%$, LDL: $+11.1 \%$, HDL: $+7.1 \%$, TG: + $23.8 \%$ ) was consistent with the results of a smaller observational study of 238 PLWH switching from TDF to TAF, which reported statistically significant relative increases in CHOL, LDL and HDL of a similar magnitude to those 
reported here [19-22]. However, other observational studies reported larger statistically significantly increases in lipids for up to 6 months after switch [18]. Nevertheless, the statistically significant increases in lipid levels reported in this OPERA study are consistent with the literature comparing lipid changes with TAF compared to TDF [15-17, 28-31]. This OPERA study did not focus on other surrogates of dyslipidemia, including the $\mathrm{CHOL} / \mathrm{HDL}$ ratio, as this measure does not form part of the ATPIII guideline grading for severity of dyslipidemia, nor does it form a target for treatment initiation or treatment response for dyslipidemia within the ATPIII guidelines. Of note, studies that estimated changes in $\mathrm{CHOL} / \mathrm{HDL}$ ratio found only negligible differences after a switch from TDF to TAF [20-22]. However, despite the $\mathrm{CHOL} / \mathrm{HDL}$ ratio being a predictor of cardiovascular disease risk, statistically significant increases in the ASCVD risk were observed in one cohort [21], although there was no change in the D:A:D coronary heart disease score or the Framingham risk score in another [22]. In addition, the ATPIII guidelines focus on thresholds for treatment that are based on total, LDL and HDL cholesterol and treatment targets focused on LDL cholesterol, as these are most relevant to overall risk of CVD events.

Clinically meaningful changes in lipids were also observed in this OPERA study. Indeed, the proportion of PLWH with dyslipidemia or severe dyslipidemia increased significantly after switching from TDF to TAF, when measured with CHOL (5.1-9.5\%), LDL (15.5-22.5\%) and TG (21.2-27.0\%), although it decreased when measured with HDL (34.8-30.2\%). Overall, these patterns of dyslipidemia are concerning for increased potential to adversely affect the risk of cardiovascular disease. These data are consistent with a smaller observational study of 194 PLWH switching from any non-TAF-containing regimen to a TAF-containing regimen: the proportion with dyslipidemia increased statistically significantly after switch, with a worsening of CHOL (pre switch: $4.5 \%$, post-switch: $15.5 \%, p=0.012$ ) and LDL (pre-switch: $26.4 \%$, post-switch: $42.3 \%, p=0.003$ ) [32]. In this study, increases in lipids after switch from TDF to TAF did not appear to be driven by boosting agent use. However, despite the fact that switching from ritonavir to cobicistat has been associated with a decrease in TG at 24 weeks [33], the stratified analyses did not distinguish between boosting with ritonavir versus cobicistat; as ritonavir represented only $10 \%$ of boosted regimens, it was insufficient for a stratified analysis by individual boosting agent.

Taken together, these data support the hypothesis that switching to a TAF-containing regimen could have a clinically relevant impact on cardiovascular health. Risks and benefits of switching from TDF to TAF must be weighed prior to switch. Risks to the kidney and bones with TDF (e.g., renal tubular dysfunction, bone mineral density) [2,
3] must be balanced against cardiovascular risks with TAF (e.g., dyslipidemia, weight gain) [32, 34-36]. The patient's history, comorbidities and other risk factors must therefore be considered in the decision-making process, and regular screening may be advised.

An elevated risk of ASCVD (10-year ASCDVD risk $\geq$ $7.5 \%$ ) was identified in $29 \%$ of PLWH before switch and $31 \%$ after switch to TAF. These estimates are consistent with the prevalence of 10 -year ASCVD risk $\geq 7.5 \%$ in other studies, ranging from 24 to $66 \%$ [37-43]. While this increase in the proportion of PLWH with an elevated ASCVD risk may not be clinically meaningful, it remains that close to one-third of the study population was considered at-risk, and one-fifth were at-risk and untreated. Although statin prescription increased after switch in this study, an estimated $59 \%$ of PLWH with an elevated 10-year ASCVD risk who could have benefited from treatment did not receive a statin prescription at any point while on TAF. This is particularly concerning given that the Pooled Cohort Equations calculator may underestimate risk among PLWH due to non-traditional risk factors driving their ASCVD risk [44]. Estimates of statin underutilization among PLWH meeting various guideline criteria for therapy vary greatly based on the population studied, ranging from 19 to $70 \%$ [37, 39, 40, 43]. The important variation in the proportion of PLWH who would benefit from lipid-lowering therapy and statin utilization derives from the use of different criteria in each study. Despite variations in the magnitude of lipid changes across regimens, statin use may be of benefit regardless of ART regimen used. In addition, lifestyle changes such as diet and exercise are also key elements to focus on to improve cardiovascular health that must be considered in addition to discussions about lipid-lowering agent use.

A large, diverse, and representative cohort of PLWH in care in the USA was used to identify 6451 PLWH who switched directly from TDF to TAF. In the OPERA cohort, clinical diagnoses, prescriptions, and laboratory results are captured prospectively from EMRs for all patients receiving healthcare at participating sites, providing complete and accurate clinical information reflecting real-world clinical practices. An important design element was the implementation of a self-controlled study. Lipid levels were compared within PLWH before and after switch, thus controlling for important risk factors for dyslipidemia such as family history or lifestyle, which are often unavailable in EMRs. Analyses restricted to PLWH who maintained all other ARVs as well as analyses stratified by boosted regimen use before and after switch confirmed that the lipid changes observed were not solely driven by boosting agents or other ARVs.

Despite these strengths, this analysis does have limitations. While changes in lipids after a switch from TDF to TAF were observed, this study could not control for a 
potential upward trend in lipids that may have occurred without switch due to the absence of a comparison group of PLWH remaining on TDF. Moreover, these analyses could not determine whether the changes observed were related to the removal of TDF or to the initiation of TAF. There is some evidence that switching to TAF may result in significantly increased CHOL and LDL values if switching from TDF, but may lead to decreased values if switching from a non-TDF-containing regimen [45], although an observational study reported statistically significantly higher CHOL, LDL and HDL levels with TAF compared to any other backbones [32]. Yet, the mechanism through which TAF would induce dyslipidaemia remains unknown.

This is a purely descriptive study without control for confounding (e.g., changes in antihypertensive use, change in smoking status, diet, and exercise) or the role of viral suppression, beyond the level of control achieved with the self-control design, study population restrictions, and stratifications. These findings were limited to the short-term impact of switching from TDF to TAF, using the first lipid panel performed at least 7 days after switch. In addition, the analysis was not restricted to fasting lipids as fasting status is variable in routine outpatient clinical care and either random or fasting lipid profiles are now recommended in guidelines [24, 46]. Integrase strand transfer inhibitors (INSTIs) have become the preferred third agent in the USA, which may have been better reflected with an extended inclusion period; at the time of the study (2015-2018), INSTIs were included in $47 \%$ of the TDF-containing regimens and $65 \%$ of the TAF-containing regimens. However, PIs and nonnucleoside reverse transcriptase inhibitors remain in use among ART-experienced PLWH such as those included in this study population.

While this study offers insight on missed opportunities for statin therapy in the context of a change in ART regimen, the analysis was limited to elevated 10-year ASCVD risk only and did not examine CVD outcomes; thus, not accounting for other high-risk groups defined in clinical guidelines [23]. Additionally, PLWH with pre-existing CVD events were not excluded in the evaluation of statin use by ASCVD risk. Moreover, the Pooled Cohort Equations calculator used to estimate the 10-year ASCVD risk was developed for a certain range of ages (40-79 years), systolic blood pressure (90-200 mmHg), HDL (20-100 mg/dL), and CHOL (130-320 mg/dL) [26]. Therefore, imputation of the score for individuals falling outside of these ranges may have led to some misclassification. Finally, validation studies in HIV cohorts have found that the Pooled Cohort Equation underestimated the ASCVD risk in PLWH [47]. Therefore, the number of PLWHs who would benefit from statin therapy is likely an underestimate.

\section{Conclusion}

In this large cohort of PLWHs who switched from TDF to TAF, switching was associated with an increase in the prevalence of clinically relevant dyslipidemia. Differences were not driven by boosted regimens or other ARVs. Risks associated with both TDF and TAF use must be weighed when deciding which agent is preferable for each person. Clinical interactions around the time of a regimen change represent an opportunity to intervene when PLWH present with an elevated risk of ASCVD. However, statin prescription remained suboptimal in this population.

Acknowledgments This research would not be possible without the people living with HIV in the OPERA Observational Database and the healthcare providers who care for them. Additionally, we are grateful for the following individuals: Robin Beckerman (SAS programming), Jeff Briney (QA), Bernie Stooks (Database Mgmt), Judy Johnson (Med Terminology Classification), Rodney Mood (Site Support).

\section{Declarations}

Funding This work was supported by Merck Sharp \& Dohme Corp., a subsidiary of Merck \& Co., Inc., Kenilworth, NJ, USA.

Conflicts of interest LB, JSF and GPF are employees of Epividian, Inc. Epividian has had research funded by Merck \& Co., ViiV Healthcare, Janssen Pharmaceuticals, Gilead Sciences, and AIDS Healthcare Foundation. PM has received research grants from ViiV Healthcare, Gilead Sciences and Janssen Cilag; speaker honoraria from ViiV Healthcare, Gilead Sciences, Janssen Cilag, BMS. and MSD; and advisory board participation from ViiV Healthcare, Gilead Sciences, Janssen Cilag, BMS and MSD. MBW has participated in post-conference advisory boards for the Conference on Retroviruses and Opportunistic Infections (CROI) and International AIDS Conference (IAC) and also serves as a principal investigator on ViiV Healthcare clinical trials but does not receive personal compensation for this work, which goes directly to the AIDS Healthcare Foundation. MBW is also a member of the Epidemiology and Clinical Advisory Board for Epividian. AB and GP are employees of Merck Sharp \& Dohme Corp., a subsidiary of Merck \& Co., Inc.

Ethics approval OPERA ${ }^{\circledR}$ complies with all HIPAA and HITECH requirements and receives annual institutional review board approval by Advarra IRB, including a waiver of informed consent and authorization for use of protected health information.

Consent to participate Not applicable

Consent for publication Not applicable

Availability of data and material The datasets generated and analyzed during the current study are not publicly available due to their proprietary nature, but access may be granted with parties agreeing to privacy restrictions and technological specifications and requirements.

Code availability Access to codes may be granted upon request with parties agreeing to privacy restrictions and technological specifications and requirements. 
Authors' contributions LB, PM, JSF, GP and AB contributed to the study conception and design. JSF, MBW and GPF contributed to the acquisition of data. LB performed the statistical analyses. All authors contributed to the interpretation of results. LB wrote the first draft of the manuscript. All authors read and approved the final manuscript.

Open Access This article is licensed under a Creative Commons Attribution-NonCommercial 4.0 International License, which permits any non-commercial use, sharing, adaptation, distribution and reproduction in any medium or format, as long as you give appropriate credit to the original author(s) and the source, provide a link to the Creative Commons licence, and indicate if changes were made. The images or other third party material in this article are included in the article's Creative Commons licence, unless indicated otherwise in a credit line to the material. If material is not included in the article's Creative Commons licence and your intended use is not permitted by statutory regulation or exceeds the permitted use, you will need to obtain permission directly from the copyright holder. To view a copy of this licence, visit http://creativecommons.org/licenses/by-nc/4.0/.

\section{References}

1. Panel on Antiretroviral Guidelines for Adults and Adolescents. Guidelines for the Use of Antiretroviral Agents in HIV-1-Infected Adults and Adolescents. Department of Health and Human Services. 2018. https://aidsinfo.nih.gov/contentfiles/lvguidelines/ adultandadolescentgl.pdf. Accessed 25 Oct 2018.

2. Cooper R, Wiebe N, Smith N, Keiser P, Naicker S, Tonelli M. Systematic review and meta-analysis: renal safety of tenofovir disoproxil fumarate in HIV-infected patients. Clin Infect Dis. 2010;51(5):496-505. https://doi.org/10.1086/655681.

3. Hemkens LG, Ewald H, Santini-Oliveira M, Buhler JE, Vuichard $\mathrm{D}$, Schandelmaier $\mathrm{S}$, et al. Comparative effectiveness of tenofovir in treatment-naive HIV-infected patients: systematic review and meta-analysis. HIV Clin Trials. 2015;16(5):178-89. https://doi. org/10.1179/1945577115Y.0000000004.

4. Grant PM, Cotter AG. Tenofovir and bone health. Curr Opin HIV AIDS. 2016;11(3):326-32. https://doi.org/10.1097/COH.00000 00000000248

5. Pilkington V, Hughes SL, Pepperrell T, McCann K, Gotham D, Pozniak AL, et al. Tenofovir alafenamide vs. tenofovir disoproxil fumarate: an updated meta-analysis of 14894 patients across 14 trials. AIDS. 2020;34(15):2259-68. https://doi.org/10.1097/qad. 0000000000002699

6. Tungsiripat M, Kitch D, Glesby MJ, Gupta SK, Mellors JW, Moran L, et al. A pilot study to determine the impact on dyslipidemia of adding tenofovir to stable background antiretroviral therapy: ACTG 5206. AIDS. 2010;24(11):1781-4. https://doi.org/ 10.1097/QAD.0b013e32833ad8b4.

7. Randell PA, Jackson AG, Zhong L, Yale K, Moyle GJ. The effect of tenofovir disoproxil fumarate on whole-body insulin sensitivity, lipids and adipokines in healthy volunteers. Antivir Ther. 2010;15(2):227-33. https://doi.org/10.3851/IMP1518.

8. Santos JR, Saumoy M, Curran A, Bravo I, Llibre JM, Navarro J, et al. The lipid-lowering effect of tenofovir/emtricitabine: a randomized, crossover, double-blind placebo-controlled trial. Clin Infect Dis. 2015;61(3):403-8. https://doi.org/10.1093/cid/civ296.

9. Gallant JE, Staszewski S, Pozniak AL, et al. Efficacy and safety of tenofovir df vs stavudine in combination therapy in antiretroviral-naive patients: a 3-year randomized trial. JAMA. 2004;292(2):191-201. https://doi.org/10.1001/jama.292.2.191.

10. Behrens G, Maserati R, Rieger A, Domingo P, Abel F, Wang $\mathrm{H}$, et al. Switching to tenofovir/emtricitabine from abacavir/ lamivudine in HIV-infected adults with raised cholesterol: effect on lipid profiles. Antivir Ther. 2012;17(6):1011-20. https://doi. org/10.3851/IMP2305.

11. Crane HM, Grunfeld C, Willig JH, Mugavero MJ, Van Rompaey $\mathrm{S}$, Moore R, et al. Impact of NRTIs on lipid levels among a large HIV-infected cohort initiating antiretroviral therapy in clinical care. AIDS. 2011;25(2):185-95. https://doi.org/10.1097/QAD. 0b013e328341f925.

12. Glidden DV, Mulligan K, McMahan V et al. Metabolic effects of preexposure prophylaxis with coformulated tenofovir disoproxil fumarate and emtricitabine. Clin Infect Dis. 2018;67(3):411-9.

13. Shaheen AA, AlMattooq M, Yazdanfar S, Burak KW, Swain MG, Congly SE, et al. Tenofovir disoproxil fumarate significantly decreases serum lipoprotein levels compared with entecavir nucleos(t)ide analogue therapy in chronic hepatitis B carriers. Aliment Pharmacol Ther. 2017;46(6):599-604. https://doi.org/ 10.1111/apt.14218.

14. De Clercq E. Tenofovir alafenamide (TAF) as the successor of tenofovir disoproxil fumarate (TDF). Biochem Pharmacol. 2016;119:1-7. https://doi.org/10.1016/j.bcp.2016.04.015.

15. Orkin C, Castelli F, Yazdanpanah Y, Rockstroh J, Brinson C, DiPerri G et al. PEB104. Cardiovascular disease risk assessments and fasting lipid changes in virologically suppressed patients randomized to switch to tenofovir alafenamide versus continuing tenofovir disoproxil fumarate. In: 22nd International AIDS Conference; 23-27 July; Amsterdam, the Netherlands 2018.

16. Raffi F, Orkin C, Clarke A, Slama L, Gallant J, Daar E, et al. Brief report: long-term (96-Week) efficacy and safety after switching from tenofovir disoproxil fumarate to tenofovir alafenamide in HIV-infected, virologically suppressed adults. J Acq Immune Def Syndr (1999). 2017;75(2):226-31. https://doi.org/10.1097/QAI. 0000000000001344.

17. Mills A, Arribas JR, Andrade-Villanueva J, DiPerri G, Van Lunzen J, Koenig E, et al. Switching from tenofovir disoproxil fumarate to tenofovir alafenamide in antiretroviral regimens for virologically suppressed adults with HIV-1 infection: a randomised, active-controlled, multicentre, open-label, phase 3, noninferiority study. Lancet Infect Dis. 2016;16(1):43-52. https://doi. org/10.1016/S1473-3099(15)00348-5.

18. Gazzola L, Tagliaferri G, Mondatore D, De Bona A, Borsino C, Bini $\mathrm{T}$, et al. Increases in lipid profile after switch from TDF to TAF-based HAART regimens in a cohort of HIV-positive patients: is it clinically relevant? J Int AIDS Soc. 2018;21(Suppl 8):e25187. https://doi.org/10.1002/jia2.25187.

19. Ewers E, Won S, Okulicz J, Ferguson T, Deiss R, Maves R, et al. 2248. Changes in lipid profiles for patients to tenofovir alafenamide (TAF)-containing regimens: perspectives from a military HIV-positive cohort. Open Forum Infect Dis. 2018;5(Suppl 1):S665-S. https://doi.org/10.1093/ofid/ofy210.1901.

20. Milinkovic A, Berger F, Arenas-Pinto A, Mauss S. Reversible effect on lipids by switching from tenofovir disoproxil fumarate to tenofovir alafenamide and back. AIDS. 2019;33(15):2387-91. https://doi.org/10.1097/qad.0000000000002350.

21. Schafer JJ, Sassa KN, O'Connor JR, Shimada A, Keith SW, DeSimone JA. Changes in body mass index and atherosclerotic disease risk score after switching from tenofovir disoproxil fumarate to tenofovir alafenamide. Open Forum Infect Dis. 2019;6(10):ofz414. https://doi.org/10.1093/ofid/ofz414.

22. Squillace N, Ricci E, Menzaghi B, De Socio GV, Passerini S, Martinelli $\mathrm{C}$, et al. The effect of switching from tenofovir disoproxil fumarate (TDF) to tenofovir alafenamide (TAF) on liver enzymes, glucose, and lipid profile. Drug Des Devel Ther. 2020;14:551520. https://doi.org/10.2147/DDDT.S274307.

23. Stone NJ, Robinson JG, Lichtenstein AH, Bairey Merz CN, Blum $\mathrm{CB}$, Eckel RH, et al. 2013 ACC/AHA guideline on the treatment of blood cholesterol to reduce atherosclerotic cardiovascular risk 
in adults: a report of the American College of Cardiology/American Heart Association Task Force on Practice Guidelines. J Am Coll Cardiol. 2014;63(25, Part B):2889-934. https://doi.org/10. 1016/j.jacc.2013.11.002.

24. Panel on Antiretroviral Guidelines for Adults and Adolescents. Guidelines for the Use of Antiretroviral Agents in HIV-1-Infected Adults and Adolescents. Department of Health and Human Services. 2019. https://files.aidsinfo.nih.gov/contentfiles/lvguidelin es/AdultandAdolescentGL.pdf. Accessed 29 Apr 2020.

25. U.S. Department of Health and Human Services. Third Report of the National Cholesterol Education Program (NCEP) Expert Panel on Detection, Evaluation, and Treatment of High Blood Cholesterol in Adults (Adult Treatment Panel III). NIH Publication 2001. https://www.nhlbi.nih.gov/files/docs/guidelines/atp3x sum.pdf. Accessed 5 June 2019.

26. Goff DC, Lloyd-Jones DM, Bennett G, Coady S, D'Agostino RB, Gibbons R, et al. ACC/AHA guideline on the assessment of cardiovascular risk: a report of the American College of Cardiology/ American Heart Association Task Force on Practice Guidelines. J Am Coll Cardiol. 2014;63(25, Part B):2935-59. https://doi.org/ 10.1016/j.jacc.2013.11.005.

27. Wilson PW. Cardiovascular disease risk assessment for primary prevention: Our approach. In: Post TW, editor. UpToDate. Waltham: UpToDate; 2019.

28. Sax PE, Wohl D, Yin MT, Post F, DeJesus E, Saag M, et al. Tenofovir alafenamide versus tenofovir disoproxil fumarate, coformulated with elvitegravir, cobicistat, and emtricitabine, for initial treatment of HIV-1 infection: two randomised, double-blind, phase 3, non-inferiority trials. Lancet. 2015;385(9987):2606-15. https://doi.org/10.1016/S0140-6736(15)60616-X.

29. Arribas JR, Thompson M, Sax PE, Haas B, McDonald C, Wohl $\mathrm{DA}$, et al. Brief report: randomized, double-blind comparison of tenofovir alafenamide (TAF) vs tenofovir disoproxil fumarate (TDF), each coformulated with elvitegravir, cobicistat, and emtricitabine $(\mathrm{E} / \mathrm{C} / \mathrm{F})$ for initial HIV-1 treatment week 144 results. JAIDS J Acq Immune Def Syndr. 2017;75(2):211-8. https://doi. org/10.1097/qai.0000000000001350.

30. Orkin C, DeJesus E, Ramgopal M, Crofoot G, Ruane P, LaMarca A, et al. Switching from tenofovir disoproxil fumarate to tenofovir alafenamide coformulated with rilpivirine and emtricitabine in virally suppressed adults with HIV-1 infection: a randomised, double-blind, multicentre, phase $3 \mathrm{~b}$, non-inferiority study. The Lancet HIV. 2017;4(5):e195-204. https://doi.org/10.1016/S23523018(17)30031-0.

31. Cid-Silva P, Fernandez-Bargiela N, Margusino-Framinan L, Balboa-Barreiro V, Mena-De-Cea A, Lopez-Calvo S, et al. Treatment with tenofovir alafenamide fumarate worsens the lipid profile of HIV-infected patients versus treatment with tenofovir disoproxil fumarate, each coformulated with elvitegravir, cobicistat, and emtricitabine. Basic Clin Pharmacol Toxicol. 2019;124(4):47990. https://doi.org/10.1111/bcpt.13161.

32. Lacey A, Savinelli S, Barco EA, Macken A, Cotter AG, Sheehan $\mathrm{G}$, et al. Investigating the effect of antiretroviral switch to tenofovir alafenamide on lipid profiles in people living with HIV. AIDS. 2020;34(8):1161-70. https://doi.org/10.1097/qad.00000 00000002541.

33. Echeverria P, Bonjoch A, Puig J, Ornella A, Clotet B, Negredo E. Significant improvement in triglyceride levels after switching from ritonavir to cobicistat in suppressed HIV-1-infected subjects with dyslipidaemia. HIV Med. 2017;18(10):782-6. https://doi. org/10.1111/hiv.12530.

34. Mallon PW, Brunet L, Hsu RK, Fusco JS, Mounzer KC, Prajapati $\mathrm{G}$, et al. Weight gain before and after switch from TDF to TAF in a U.S. cohort study. J Int AIDS Soc. 2021;24(4):e25702. https:// doi.org/10.1002/jia2.25702.
35. Surial B, Mugglin C, Calmy A, Cavassini M, Gunthard HF, Stockle M, et al. Weight and metabolic changes after switching from tenofovir disoproxil fumarate to tenofovir alafenamide in people living with HIV : a cohort study. Ann Intern Med. 2021. https://doi.org/10.7326/M20-4853.

36. Łomiak M, Stępnicki J, Mikuła T, Wiercińska-Drapało A. Weight and body mass index increase after switch from tenofovir disoproxil fumarate to tenofovir alafenamide fumarate-containing treatment in an antiretroviral therapy-experienced group. Int J STD \& AIDS. 2021. https://doi.org/10.1177/0956462420983699.

37. Kelly SG, Krueger KM, Grant JL, Penugonda S, Feinstein MJ, Taiwo BO, et al. Statin prescribing practices in the comprehensive care for HIV-infected patients. JAIDS J Acq Immune Def Synd. 2017;76(1):e26-9. https://doi.org/10.1097/qai.0000000000001454.

38. Begovac J, Dragovic G, Viskovic K, Kusic J, Perovic Mihanovic M, Lukas D, et al. Comparison of four international cardiovascular disease prediction models and the prevalence of eligibility for lipid lowering therapy in HIV infected patients on antiretroviral therapy. Croat Med J. 2015;56(1):14-23.

39. Clement ME, Park LP, Navar AM, Okeke NL, Pencina MJ, Douglas PS, et al. Statin utilization and recommendations among HIV- and HCV-infected veterans: a cohort study. Clin Infect Dis. 2016;63(3):407-13. https://doi.org/10.1093/cid/ciw289.

40. Bagchi S, Patel P, Faramand R, Burrowes S, Hossain MB, Kottilil $S$, et al. Underutilization of statins for prevention of cardiovascular disease among primarily African-American HIV-infected patients. J AIDS Clin Res. 2015. https://doi.org/10.4172/2155-6113.10004 99.

41. Todd JV, Cole SR, Wohl DA, Simpson RJJ, Jonsson Funk M, Brookhart MA, et al. Underutilization of statins when indicated in HIV-seropositive and seronegative women. AIDS Patient Care STDS. 2017;31(11):447-54. https://doi.org/10.1089/apc.2017. 0145 .

42. Lichtenstein KA, Armon C, Buchacz K, Chmiel JS, Buckner K, Tedaldi E, et al. Provider compliance with guidelines for management of cardiovascular risk in HIV-infected patients. Prev Chronic Dis. 2013;10:E10. https://doi.org/10.5888/pcd10.120083.

43. Reinsch N, Neuhaus K, Esser S, Potthoff A, Hower M, Mostardt $\mathrm{S}$, et al. Are HIV patients undertreated? Cardiovascular risk factors in HIV: results of the HIV-HEART study. Eur J Prev Cardiol. 2012;19(2):267-74. https://doi.org/10.1177/1741826711398431.

44. Zanni MV, Fitch KV, Feldpausch M, Han A, Lee H, Lu MT, et al. 2013 American College of Cardiology/American Heart Association and 2004 adult treatment panel III cholesterol guidelines applied to HIV-infected patients with/without subclinical high-risk coronary plaque. AIDS (London, England). 2014;28(14):206170. https://doi.org/10.1097/QAD.0000000000000360.

45. Pozniak A, Arribas JR, Gathe J, Gupta SK, Post FA, Bloch M, et al. Switching to tenofovir alafenamide, coformulated with elvitegravir, cobicistat, and emtricitabine, in HIV-infected patients with renal impairment: 48-week results from a single-arm, multicenter, open-label phase 3 study (1999). J Acq Immune Def Syndr. 2016;71(5):530-7. https://doi.org/10.1097/QAI.00000 00000000908 .

46. Grundy SM, Stone NJ, Bailey AL, Beam C, Birtcher KK, Blumenthal RS, et al. 2018 AHA/ACC/AACVPR/AAPA/ABC/ACPM/ ADA/AGS/APhA/ASPC/NLA/PCNA guideline on the management of blood cholesterol: executive summary: a report of the American College of Cardiology/American Heart Association Task Force on Clinical Practice Guidelines. J Am Coll Cardiol. 2019;73(24):3168-209. https://doi.org/10.1016/j.jacc.2018.11.002.

47. Achhra AC, Lyass A, Borowsky L, Bogorodskaya M, Plutzky J, Massaro JM, et al. Assessing cardiovascular risk in people living with HIV: current tools and limitations. Curr HIV/AIDS Rep. 2021;18(4):271-9. https://doi.org/10.1007/s11904-021-00567-w. 